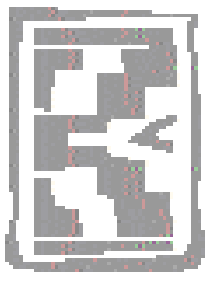

\title{
The heart remembers: observations of cardiac memory in the Dorper sheep heart
}

\author{
J. KER ${ }^{1}$, E.C. WEBB ${ }^{2}$, J.A. KER ${ }^{3}$ and P.A. BEKKER ${ }^{1}$
}

\begin{abstract}
J. KER, E.C. WEBB, J.A. KER \& P.A. BEKKER. 2003. The heart remembers: observations of cardiac memory in the Dorper sheep heart. Onderstepoort Journal of Veterinary Research, 70:299-305

Memory is a property common to a diverse range of tissues. Cardiac memory has been demonstrated in the human, dog, rat and rabbit. This is a peculiar phenomenon, reflected in the T wave of the electrocardiogram. The heart is able to remember periods of alterations in the sequence of ventricular activation and once there is a return to a normal sequence of ventricular activation the $T$ waves may manifest memory. Cardiac memory is noted when the T wave during normal ventricular activation retains the vector of the previous abnormal QRS complex, caused by a period of altered ventricular activation. Possible mechanisms of memory in the heart are alterations of the transient outward potassium current $\left(I_{\text {to }}\right)$ in ventricular myocytes and new protein synthesis inside myocytes. These two mechanisms operate in short- and long-term cardiac memory respectively. Currently, it is unknown whether memory may have adverse structural consequences in the heart. We were able to demonstrate memory in the hearts of Dorper wethers and this is the first report of cardiac memory in Dorper sheep.

Keywords: Cardiac memory, Dorper sheep, electrocardiograph
\end{abstract}

\section{INTRODUCTION}

Memory is a property common to a diverse range of tissues, such as the brain and immune system (Herweg \& Chang 2001; Rosen 2001), but is it possible for the heart to remember? Indeed, this appears to be the case-cardiac memory has been demonstrated in the heart of the human, dog, rat and rabbit (Rosenbaum \& Blanco 1982; Goldberger \& Kadish 1999; Herweg \& Chang 2001; Rosen 2001, 2002).

\footnotetext{
Department of Physiology, Faculty of Medicine, University of Pretoria, P.O. Box 24318, Gezina, Pretoria, 0031 South Africa. E-mail: james.ker@med.up.ac.za

2 Department of Animal and Wildlife Sciences, Faculty of Agricultural Sciences, University of Pretoria, Pretoria, 0002 South Africa

3 Department of Internal Medicine, Faculty of Medicine, University of Pretoria, Pretoria, 0002 South Africa

Accepted for publication 11 June 2003-Editor
}

Cardiac memory is manifested as peculiar changes of the T wave, seen on the electrocardiogram (Rosenbaum \& Blanco 1982; Goldberger \& Kadish 1999; Rosen 2001, 2002). There are three waveforms on an electrocardiogram (ECG). The $P$ wave reflects atrial activation, the QRS complex ventricular activation, and the $T$ wave ventricular repolarization (Rosen 2001). The $T$ wave actually reflects both transmural and apico-basal gradients for ventricular repolarization. Ultimately, $\mathrm{T}$ waves derive from a balance between the inward and outward ion currents that occur in individual ventricular myocytes (Rosen 2001, 2002). The factors affecting $T$ wave expression are regional differences in these ionic currents with the action potentials induced by them and the temporal sequence of ventricular activation (Rosen 2001). The consequence is voltage gradients in the ventricle, both transmurally and apico-basally (Rosen 2001). But how is cardiac memory manifested in the T wave and what type of 
stimuli is considered worthy of remembrance by the heart?

Only one event is remembered by the heart and that is a period (or periods) of altered ventricular activation (Rosenbaum \& Blanco 1982; Goldberger \& Kadish 1999; Rosen 2001). A variety of clinical scenarios are able to cause abnormal ventricular activation and these include: ventricular pacing, intermittent left bundle branch block, premature ventricular complexes and ventricular preexcitation (Rosenbaum \& Blanco 1982; Geller \& Rosen 1993; Nirei \& Kasanuki 1997; Geller \& Carlson 1999; Goldberger \& Kadish 1999; Sporton \& Holdright 2001).

Normally, a depolarization impulse is initiated in the atrium and reaches the ventricles by way of the specialized conduction system (Van Dam 1989). However, during one of the above situations of altered ventricular activation, ventricular activation is initiated in one of the ventricles itself and the depolarization front moves in a lateral direction to the other ventricle. It is these periods of altered (abnormal) ventricular activation that the heart may remember.

When cardiac memory is noted, the direction (polarity) of the T wave is similar to the direction (polarity) of the QRS complexes noted during the period(s) of abnormal ventricular activation (Goldberger \& Kadish 1999). Rosenbaum \& Blanco (1982), in their original description of cardiac memory, noted a specific sequence in cardiac memory. Periods of abnormal ventricular activation (an altered sequence of ventricular depolarization) may induce a change in the T wave, which will be noted after return to a normal sequence of ventricular activation. The $T$ wave will retain the vector of the previous abnormal QRS complex-the polarity or direction of this T wave will be the same as that of the abnormal QRS complex(es). We wanted to know if the Dorper sheep heart is able to manifest cardiac memory.

\section{MATERIALS AND METHODS}

This study was performed with the approval of, and adherence to, the guidelines of the Pretoria Biomedical Research Centre's Animal Use and Care Committee. Clinically normal Dorper wethers $(n=$ 6 ), all between the ages of 9 and 12 months, and weighing between 35 and $40 \mathrm{~kg}$ were used in the study. They were each fed on lucerne hay ad libitum, supplemented with $300 \mathrm{~g}$ per day of pelleted concentrate (10 MJ ME/kg DM with $14 \%$ crude protein) and had free access to water at all times.
The wethers were placed into two groups. The wethers in one of the groups $(n=4)$ were sedated with ketamine hydrochloride (Brevinaze) at a dose of $100 \mathrm{mg}$ intramuscularly once only while those in the second group $(n=2)$ were sedated with midazolam (Dormicum) at a dose of $30 \mathrm{mg}$ intramuscularly once only. They were then placed in the right lateral decubitus position. Two different sedatives were used to exclude the possibility that the choice of sedative may have electrocardiographic effects, either potentiating or inhibiting the occurrence of cardiac memory. After an interval of 10 min there were no spontaneous movements and baseline electrocardiographs (ECGs) were obtained.

\section{Method of electrocardiography}

- Einthoven's triangle was moved from the frontal to the sagittal plane, as described before by Schultz \& Pretorius (1972), by moving the standard and unipolar limb electrodes as follows:

(a) aVR moved from the right fore limb to the head between the ears;

(b) aVL moved from the left fore limb to the sacrum;

(c) aVF moved from the left hind limb to the sternal angle; and

(d) the earth electrode was placed on the right hind leg, just above the hock.

- The six precardial leads were placed as follows:

(a) V1 placed $7 \mathrm{~cm}$ to the right of the sternal angle;

(b) V2 placed $7 \mathrm{~cm}$ to the left of the sternal angle;

(c) V3 placed $4.5 \mathrm{~cm}$ below and $1 \mathrm{~cm}$ to the left of $\mathrm{V} 2$;

(d) V4 placed $4.5 \mathrm{~cm}$ below and $1 \mathrm{~cm}$ to the left of V3;

(e) V5 placed $4.5 \mathrm{~cm}$ below and $1 \mathrm{~cm}$ to the left of $\mathrm{V} 4$; and

(f) V6 placed $4.5 \mathrm{~cm}$ below and $1 \mathrm{~cm}$ to the left of V5.

- Meditrace 200, disposable ECG conductive, adhesive electrodes were used. The skin areas where ECG electrodes were placed were shaven and the electrodes were secured with Super Glue (Bostik).

- A 12 lead electrocardiogram was performed with a Schiller AT-2 plus six channel electrocardiograph. The paper speed was set at $25 \mathrm{~mm} / \mathrm{s}$. 
We induced premature ventricular complexes (PVCs) to alter the normal sequence of ventricular activation by positioning a spring-wire guide on the endocardial aspect of the right ventricle via the left internal jugular vein, utilizing the Seldinger technique. The position of the spring-wire guide was confirmed by X-ray and mechanical movement of the wire produced premature ventricular complexes.

\section{RESULTS}

Cardiac memory was demonstrated in five of the six Dorper wethers and memory $T$ waves were seen in leads III and V1 only. Lead III proved to be the most useful lead to demonstrate cardiac memory as memory $T$ waves were seen in all the cases in this lead while only one wether demonstrated memory $\mathrm{T}$ waves in lead $\mathrm{V} 1$.

FIG. 1 Example 1-cardiac memory, demonstrated

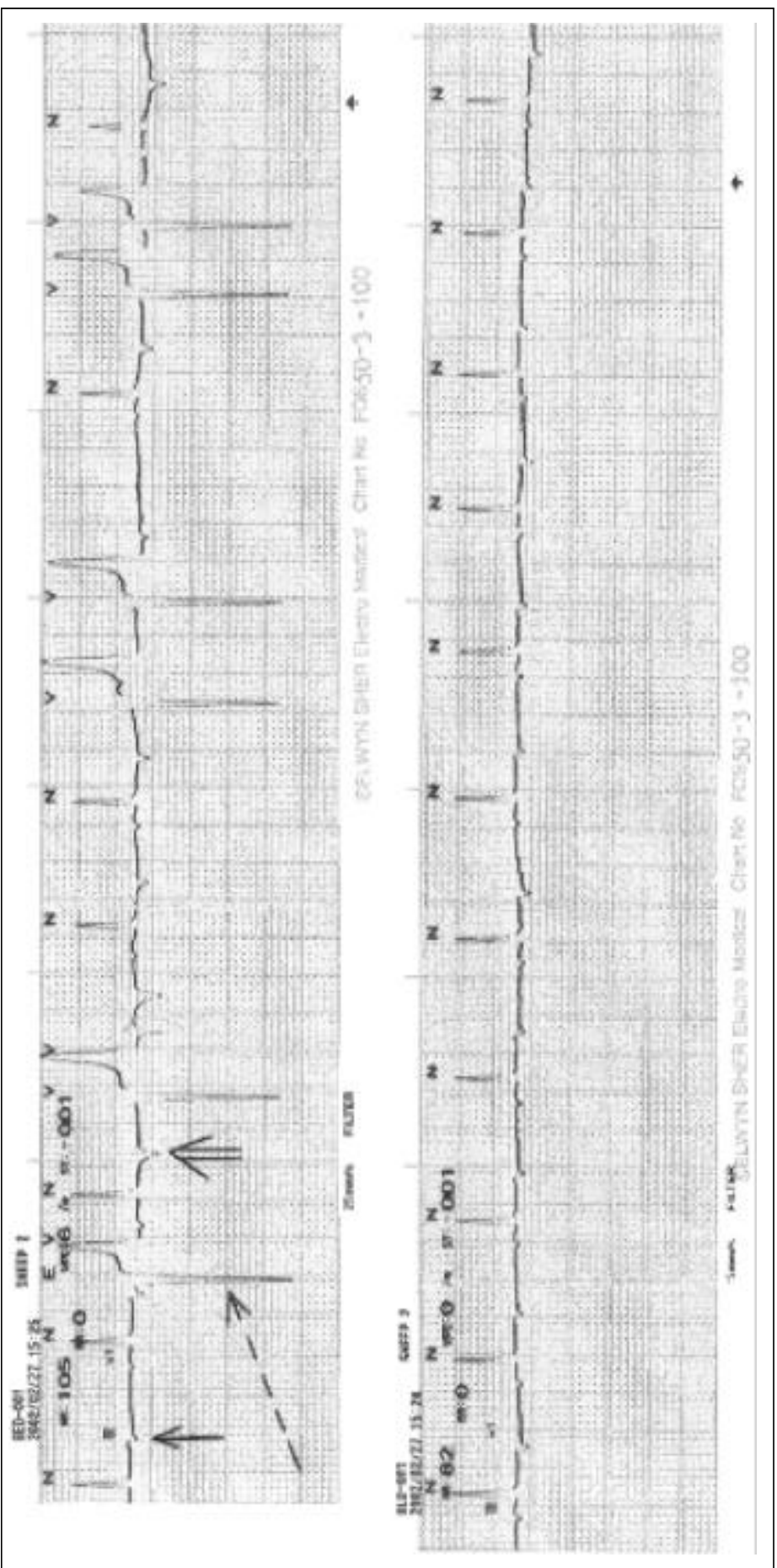




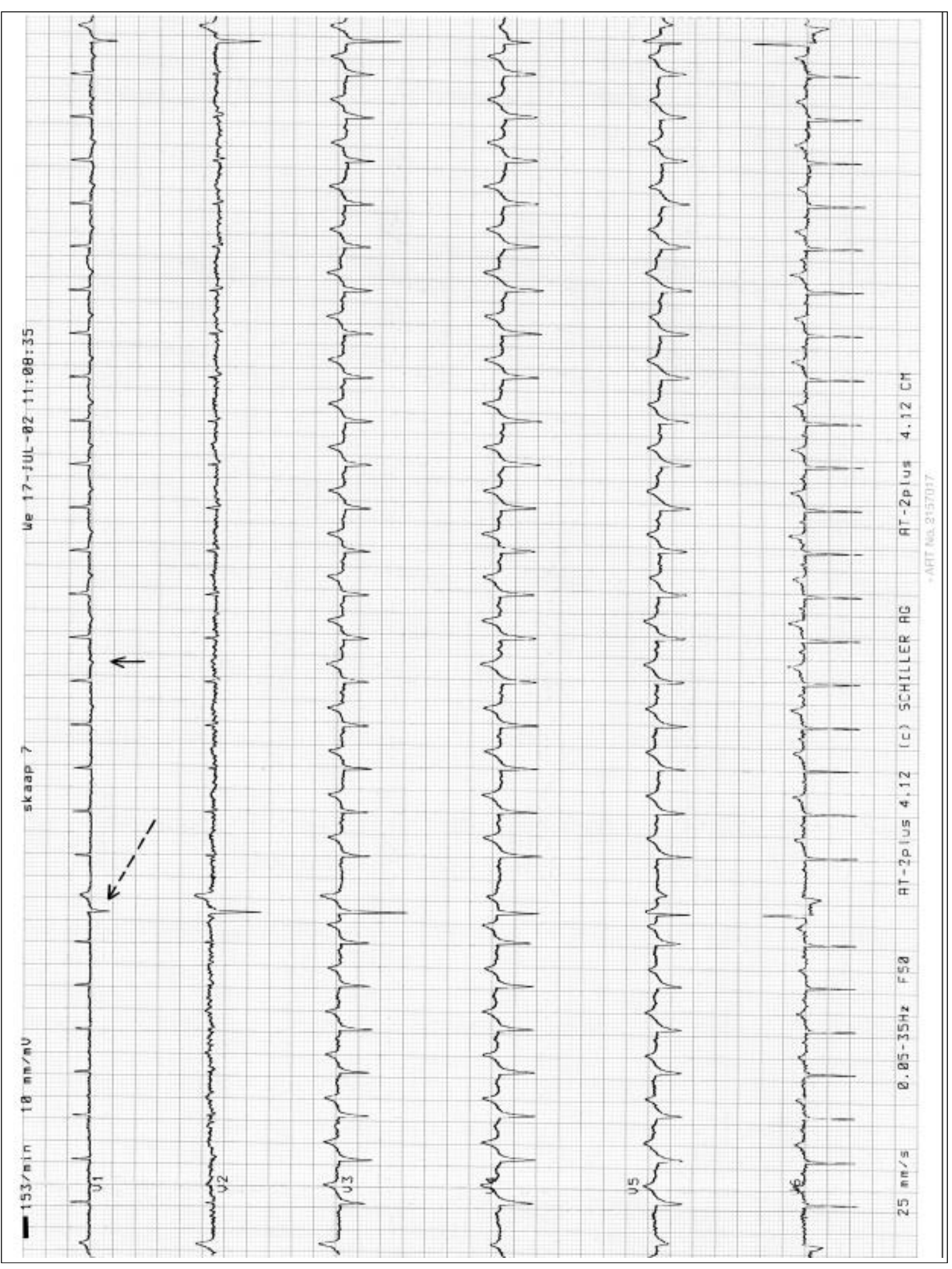

FIG. 2 Example 2-cardiac memory, demonstrated in lead VI 


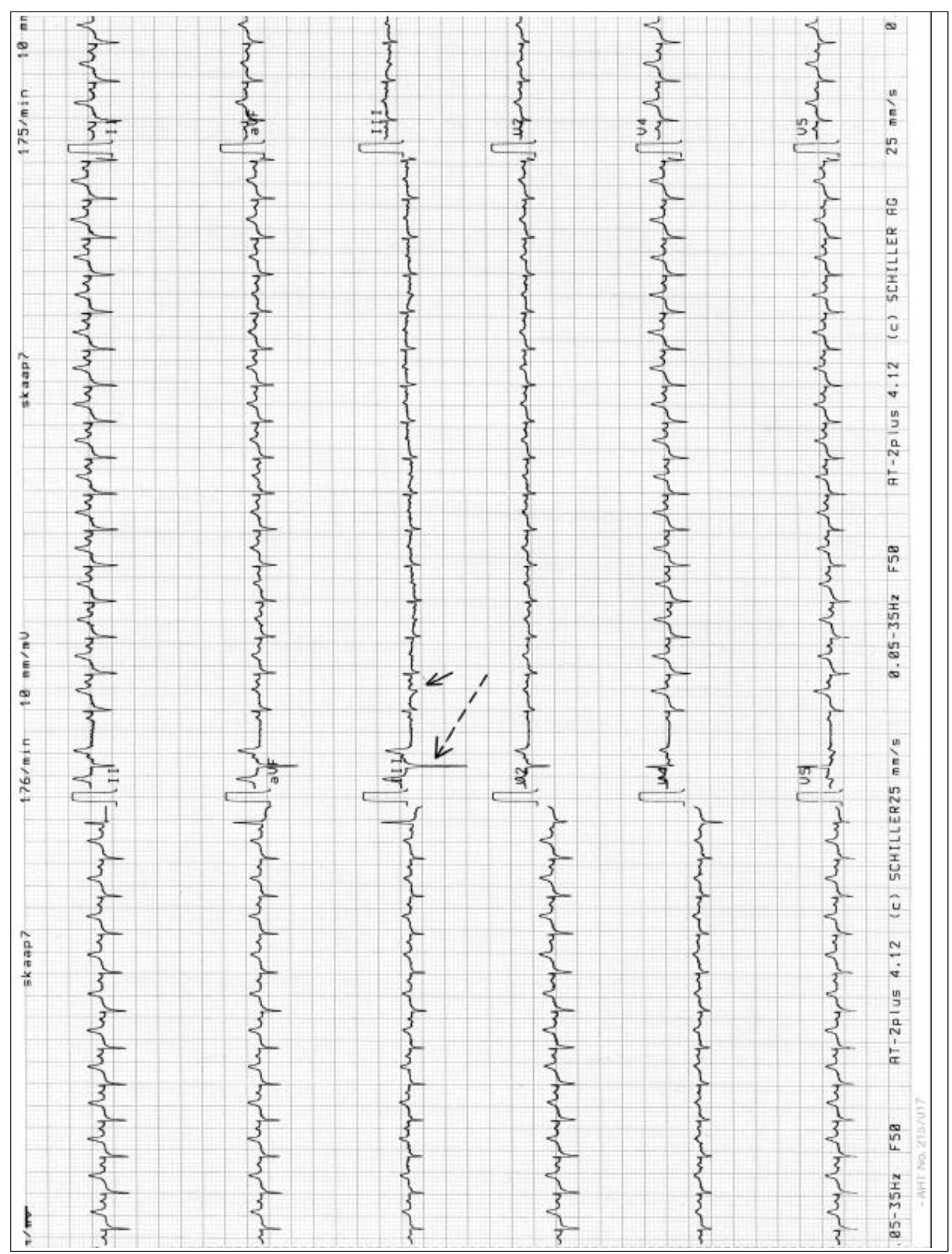

FIG. 3 Example 3-cardiac memory, demonstrated in lead III 
Fig. 1 demonstrates an example from an ECG of one of the Dorper wethers. The first two beats are normal sinus beats. The $\mathrm{T}$ waves are biphasic (arrow). The third beat is a premature ventricular complex (broken arrow). Note that the polarity of the QRS complex of the PVC differs from that of the normal complexes-the QRS complex of the normal beats are positive while that of the PVC complexes are negative. The fourth beat is the first normal beat after the PVC. The T wave of this beat is negative - the polarity of this $T$ wave follows the polarity of the QRS complex of the PVC-the heart "remembers". This memory $\mathrm{T}$ wave is indicated by the double arrow.

In Fig. 2 an example of cardiac memory in lead V1 is illustrated, and in Fig. 3 an example of cardiac memory in lead III is shown. The conventions regarding labeling and arrows are the same as for Fig. 1.

\section{DISCUSSION}

Cardiac memory T waves may develop after either short or long periods of altered ventricular activation and is called short-term and long-term cardiac memory respectively (Goldberger \& Kadish 1999; Rosen 2001). However, there is no consensus yet on the period of time required to separate shortfrom long-term cardiac memory (Goldberger \& Kadish 1999). Rosenbaum \& Blanco (1982) in the first cardiac memory experiments needed 15 min of right ventricular pacing to demonstrate memory $T$ waves. Goyal \& Syed (1998) were recently able to observe cardiac memory after only $1 \mathrm{~min}$ of right ventricular pacing in humans.

$\mathrm{T}$ wave changes may occur under a variety of circumstances (Rosen 2001). For many years it was thought that all $\mathrm{T}$ wave changes may be explained by Wilson's formulation (Levine \& Lown 1952; Rosenbaum \& Blanco 1982; Rosen 2001). This classic formulation states that all $\mathrm{T}$ wave changes are either primary or secondary in nature (Wilson \& Macleod 1931). A primary T wave change is any change in the polarity, amplitude or duration of a $T$ wave, where the QRS complex preceding the altered T wave is normal (Levine \& Lown 1952; Rosenbaum \& Blanco 1982; Rosen 2001). Therefore, primary T wave changes are independent of the QRS complex and are derived from intrinsic ventricular ion channels and other electrical determinants of ventricular repolarization (Rosen 2001). Secondary T wave changes, on the other hand, derive from an altered sequence of ventricular activation-the preceding QRS complex must be abnormal (Rosen- baum \& Blanco 1982; Rosen 2001) and these T wave changes are seen during the period of abnormal ventricular activation. With the discovery of cardiac memory there came a third type of $T$ wave change - the pseudoprimary $\mathrm{T}$ wave change (Rosen 2001). T wave changes caused by memory are not secondary, because the preceding QRS complex is normal-memory is only seen during reversion to normal sinus rhythm (Rosenbaum \& Blanco 1982; Rosen 2001, 2002). They are also not primary, as they were preceded by a period of altered ventricular activation, and are therefore classified as "pseudoprimary".

The mechanisms of cardiac memory involve alterations in ventricular ion channels and the synthesis of new proteins (Rosen 2001, 2002). In the induction of short-term cardiac memory, both the transient outward potassium channel $\left(I_{t o}\right)$ and the hormone angiotensin II play an integral role, because if the $\mathrm{I}_{\text {to }}$, or the receptors for angiotensin II are blocked, short-term cardiac memory will not occur (Rosen 2001, 2002). Long-term cardiac memory is suppressed if the synthesis of new proteins is prevented (Shvilkin \& Danilo 1998; Rosen 2001, 2002). In the central nervous system, memory is a process of long-term potentiation, induced by repetitive exposure to a signal that results in new protein synthesis (Shvilkin \& Danilo 1998). Neuroscientists rely on cycloheximide, an inhibitor of new protein synthesis, to differentiate between these two mechanisms (Shvilkin \& Danilo 1998). A significant delay in the development of long-term cardiac memory has been observed in the dog after the administration of cycloheximide (Shvilkin \& Danilo 1998).

Currently, it is not known whether the development of cardiac memory may have any possible adverse structural consequences in the heart (Rosen 2001, 2002). On a subcellular level cardiac memory is associated with a reduction in density of the gap junctional protein connexin 43 (Cx43) and also with changes in the distribution of $\mathrm{C} \times 43$, changing from concentration at the longitudinal poles of myocytes to a more uniform distribution across the lateral margins of the cell (Patel \& Plotnikov 2001; Rosen 2002). Furthermore, these changes in Cx43 are non-uniform-greater epicardially than endocardially (Patel \& Plotnikov 2001).

The possible adverse structural effects of cardiac memory on the heart deserves further study. We propose that our ovine model of cardiac memory in the Dorper sheep heart is a valid model that may serve as the basis for further investigation of such possible cardiac structural alterations. 


\section{REFERENCES}

GELLER, J.C. \& ROSEN, M.R. 1993. Persistent T wave changes after alteration of the ventricular activation sequence. New insights into cellular mechanisms of cardiac memory. Circulation, 88:1811-1819.

GELLER, J.C. \& CARLSON, M.D. 1999. Persistent T wave changes after radiofrequency catheter ablation of an accessory connection (WPW syndrome) are caused by cardiac memory. American Heart Journal, 138:987-993.

GOLDBERGER, J.J. \& KADISH, A.H. 1999. Cardiac memory. Pace, 22:1672-1679.

GOYAL, R. \& SYED, Z. 1998. Changes in cardiac repolarization following short periods of ventricular pacing. Journal of Cardiovascular Electrophysiology, 9:269-280.

HERWEG, B. \& CHANG, F. 2001. Cardiac memory in canine atrium. Identification and implications. Circulation, 103:455461.

LEVINE, H.D. \& LOWN, B. 1952. The clinical significance of postextrasystolic T wave changes. Circulation, 6:538-548.

NIREI, T. \& KASANUKI, H. 1997. Cardiac memory in patients with intermittent Wolff-Parkinson-White syndrome. Journal of Electrocardiology, 30:323-329.
PATEL, P.M. \& PLOTNIKOV, A. 2001. Altering ventricular activation remodels gap junction distribution in canine heart. Journal of Cardiovascular Electrophysiology, 12:570-577.

ROSEN, M.R. 2001. The heart remembers: clinical implications. Lancet, 357:468-471.

ROSEN, M.R. 2002. The electrocardiogram 100 years later. Circulation, 106:2173-2179.

ROSENBAUM, M.B. \& BLANCO, H.H. 1982. Electrotonic modulation of the T wave and cardiac memory. American Journal of Cardiology, 50:213-222.

SCHULTZ, R.A. \& PRETORIUS, P.J. 1972. An electrocardiographic study of normal sheep using a modified technique. Onderstepoort Journal of Veterinary Research, 39:97-106.

SHVILKIN, A. \& DANILO, P. 1998. Evolution and resolution of long-term cardiac memory. Circulation, 97:1810-1817.

SPORTON, S. \& HOLDRIGHT, D. 2001. Case 5: Cardiac memory. Hospital Medicine, 62:498-499.

VAN DAM, R.T. 1989. Activation of the heart, in Comprehensive electrocardiology, theory and practice in health and disease, edited by P.W. MacFarlane \& T.D. Veitch Lawrie. New York: Pregamon Press.

WILSON, F.N. \& MACLEOD, A.G. 1931. The T deflection of the electrocardiogram. Transatlantic Association of American Physicians, 46:29-38. 